Instituto Internacional de Investigación y Desarrollo Tecnológico Educativo INDTEC, C.A.

DOI: https://doi.org/10.29394/Scientific.issn.2542-2987.2018.3.7.14.268-288

OAI-PMH: http://www.indteca.com/ojs/index.php/Revista Scientific/oai

\title{
Proposiciones Teóricas para la Formación de la Ciudadanía Mediadas por la Tecnología
}

\author{
Autora: Tania Margarita Martínez de Padrón \\ Universidad Latinoamericana y del Caribe, ULAC \\ tania.martinez10@gmail.com \\ Caracas, Venezuela
}

\section{Resumen}

La investigación que se presenta emerge de las connotaciones referentes a la formación de la ciudadanía mediada por la Tecnología de la Información y la Comunicación. Se parte de la concepción del ciudadano actual y la influencia que ha emergido mediante la utilidad de las distintas redes sociales, así como del Proyecto Canaima Educativo y la orientación que se les brinda a los escolares en este sentido. El objetivo general fue Generar Proposiciones Teóricas que orienten la Formación de la Ciudadanía mediada por las TIC en las escuelas de Educación Primaria de Santa Teresa del Tuy. La metodología empleada fue el paradigma cualitativo, basándose en el enfoque fenomenológico interpretativo, la cual se interesa en descubrir y entender los significados, hábitos y prácticas del ser humano. Castillo (2000: 5). El investigador se acercó al campo objeto de estudio para observar, describir e interpretar una realidad. Como instrumento se empleó la entrevista en profundidad. La información obtenida se registró en cuadros que a través de la triangulación permitió cumplir con el desarrollo de los objetivos específicos. En cuyos hallazgos prevaleció la desviación del uso de herramientas tecnológicas y cómo éstas han configurado la formación de la ciudadanía en los escolares en su comportamiento y accionar. A la vez, permitió conocer desde el abordaje tecnológico educativo, que escasamente el docente brinda orientaciones que redireccionen la formación de la ciudadanía. Asimismo, se obtuvo el objetivo planteado como lo fue las proposiciones teóricas que orienten la Formación de la Ciudadanía mediadas por la TIC.

Palabras clave: formación; ciudadanía; tic. 


\title{
Theoretical Propositions for the Citizen Formation Mediated by Technology
}

\begin{abstract}
The research presented emerges from the connotations concerning the formation of the citizenship by the information technology and communication. Be part of the conception of the current citizen and the influence that has emerged through the utility of the various social networks, as well as Canaima education project and the guidance provided to schoolchildren in this sense. The general objective was to generate propositions theoretical that guide the formation of citizenship mediated by ICT in the primary schools of Santa Teresa del Tuy. The methodology used was the qualitative paradigm, based on the interpretative phenomenological approach of Heidegger, which is interested in discovering and understanding the meanings, habits and practices of the human being. Castle (2000: 5). The researcher approached the field object of study to observe, describe and interpret a reality. As an instrument used the interview in depth. The information obtained was recorded in pictures which allowed to comply with the development of specific objectives through triangulation. On whose findings prevailed deviating from the use of technological tools and how these have formed the formation of citizenship in school children in their behavior and actions. At the same time, allowed know from educational technological approach, that sparing the teacher provides guidance that redirect the formation of citizenship. Also was the stated objective as it was the theoretical propositions that guide the formation of citizens ICT-mediated.
\end{abstract}

Keywords: training; citizenship; ict.

Date Received: 21-07-2017

Date Acceptance: 02-10-2017 


\section{Introducción}

Las Tecnologías de la Información y la Comunicación (TIC), fueron intencionalmente creadas para potenciar el desarrollo de distintas ciencias, en especial en lo educativo, a favor del proceso enseñanza y aprendizaje a través del uso de materiales, herramientas y estrategias que puedan fortalecer la teoría y la práctica dentro de las aulas en todos los niveles y modalidades, ya sean presenciales o virtuales.

En este orden de ideas, con el apoyo de las herramientas Tecnológicas dentro del campo educativo, los estudiantes tienen acceso a una extensa gama de información ofrecida por la sociedad del conocimiento en la cual el manejo adecuado de términos y conceptos de cualquier área juega un papel preponderante dentro del aprendizaje y la formación ciudadana del educando.

Cabe señalar, que en Venezuela a través del Ministerio del Poder Popular para la Educación, surge para este fin la incorporación del Proyecto Canaima Educativo, que consiste en la dotación de un computador personal portátil a cada escolar desde el segundo grado de la educación básica, este plan enriquece el papel del docente, y lo canaliza hacia la estimulación de estrategias innovadoras, hacia la planificación significativa de actividades atrayentes de las actividades de aprendizaje, así como facilita la atención individualizada al orientar de manera significativa el uso de las herramientas tecnológicas.

Sobre la base de lo antes expuesto, para el proceso de formación de ciudadanía, las TIC, Incluye una relevante pieza al proponer que los estudiantes se desarrollen en un conjunto de herramientas (conocimientocomprensión, habilidades y aptitudes, valores y disposiciones), que garantiza su participación y sensible en roles y responsabilidades que asuman a futuro. En este particular, al hablar de la formación de ciudadanía, de acuerdo con la UNESCO (citado en Morawietz, 2015), la define como:

"aprender a vivir con los demás, respetándolos", especificando 
que "la idea en la que se fundamenta la educación para la ciudadanía es que la enseñanza debe contribuir a que las personas tengan medios de interaccionar con la sociedad y participar en la toma de decisiones que desembocan en políticas sociales, culturales y económicas". "El civismo no puede limitarse a las cuestiones políticas. Es una reflexión cotidiana sobre el consumo, las modalidades de transporte y las conductas que se han de observar con respecto a los conciudadanos" (pág. 4).

A tal efecto, así como la tecnología ha resultado como un apoyo en los avances tecnológicos, se busca que este apoyo sea trasladado a la formación del ciudadano desde la escuela primaria, para así lograr mejorar ampliamente el proceso educativo y buscar la organización del país al formar mejores ciudadanos.

Al respecto, el docente debe reflexionar y entender que el uso de las TIC en el ámbito educativo es un punto clave para mejorar significativamente los resultados del proceso enseñanza aprendizaje y de formación integral del estudiantado. Asimismo, tomar en cuenta lo planteado por Ausubel (citado en Luján y Salas, 2009), en las teorías constructivistas y el desarrollo de habilidades, que al implantar estas herramientas permitirán que tanto docentes como estudiantes obtengan grandes beneficios de su uso.

Sin embargo, pese a la utilidad y uso de las tecnologías, específicamente las redes sociales como medio para interactuar en la sociedad, se ha evidenciado hoy día que, en los centros educativos, aunque cuentan con las herramientas, como las denominadas canaimitas, se resisten a la aplicación de las mismas en los programas para la formación del Ciudadano. Según Hoyuelos (2006), haciendo referencia a ello, describe que se observan en las aulas de clases de Educación Primaria que algunos profesores ponen de manifiesto su apatía por la implementación de las tecnologías actuales en la educación y han estructurado fuertes barreras evitando el desarrollo de estas herramientas al interior de diferentes centros 
educativos.

Asimismo, de acuerdo con lo antes descrito, como investigadora se ha evidenciado que los docentes continúan pretendiendo afianzar la formación de ciudadanía en los estudiantes a través de la transmisión de conocimiento suficiente y comprensivo de la historia nacional, las estructuras y procesos del gobierno y la vida política, con los modos clásicos de educación cívica, donde los estudiantes se involucran de manera vaga y tímida con sus compromisos ciudadanos.

A este particular, el autor, refleja que los estudiantes deben ser motivados a aprender ciudadanía en forma activa, que experimenten en la comunidad escolar, local y global y, particularmente, participen de una comunidad democrática a través de la utilización de las herramientas tecnológicas. A través de la cual el docente deberá mediar para que se dirija esta utilidad de manera formativa en el ciudadano y no de deformación de valores como se ha evidenciado en la cotidianidad con el uso de las redes sociales por parte de los jóvenes.

Cabe destacar, que en las escuelas de Educación Primaria del casco central de Santa teresa del Tuy, los docentes no escapan de esta problemática, ya que se evidencia que le dan poca utilidad al Proyecto Canaima Educativo para la enseñanza en los escolares y es escasa la orientación que reciben estos para incursionar en las redes del internet, así como a las distintas redes sociales, en las cuales reciben múltiples mensajes que contribuyen a deformar al ciudadano que está en formación, en los aspectos de valores sociales, de educación sexual y de identidad tanto personal como nacional.

De acuerdo con tal planteamiento, la investigadora se propuso Generar Proposiciones Teóricas que orienten la formación de Ciudadanía mediadas por la Tecnología de la Información y Comunicación en las escuelas de Educación Primaria del casco central de Santa Teresa del Tuy. Mediante las mismas, el 
docente comenzará a destacar su labor educativa implementando estrategias que conducirá a desarrollar en los educandos una nueva formación ciudadana medada por las TIC.

Sobre la base de lo anteriormente expuesto surgen las siguientes interrogantes:

¿Cuáles son las metodologías pedagógicas insertas en las Tic utilizadas por los docentes de las escuelas de educación primaria del casco central de Santa Teresa del Tuy al orientar la formación de la ciudadanía en los educandos? ¿Cómo ha sido la orientación educativa que asumen los docentes de estas escuelas de educación primaria ante la utilización de la Tecnología de la Información y Comunicación para la formación de la ciudadanía de los escolares? ¿Cuál es el significado que le asignan los docentes de las escuelas mencionadas, al uso de las TIC en el proceso de formación ciudadana en los escolares de estas escuelas? ¿Qué orientaciones pueden surgir a través de esta indagación para la generación de proposiciones teóricas en la Formación de la Ciudadanía mediante la Tecnología de la Información y la Comunicación?

\section{Fundamento Teórico}

\subsection{Concepciones de ciudadanía.}

Al realizar la concepción de ciudadanía se hace preciso revisar sus antecedentes históricos que produjo su conformación. De acuerdo con Horrach (2009), La ciudadanía, remonta su origen a la Grecia antigua, en Atenas, donde funcionaba un sistema social de jerarquía, aunque no autoritario, la ciudadanía fue evolucionando de una posición de control a un ejercicio directo del poder. El autor sostiene que se refiere a un modelo con un espíritu que consistía en desarrollar un proyecto de autonomía según el cual cada individuo fuera importante para el funcionamiento de la comunidad, de 
modo tal que ciudadanía y Estado no se diferenciaban.

Al respecto, según Rueda (2009), hoy día, la evolución de la ciudadanía se ha visto impactada significativamente con la llegada de las tecnologías de la información y la comunicación social, sin embargo, en este tiempo los ciudadanos son plenamente conscientes que con la aparición de este fenómeno no es suficiente para el desarrollo del país y de mejores ciudadanos, debido a que es inexorable contar con políticas educativas que orienten y dirijan su uso hacia una adecuada formación.

\subsection{Tecnología de la Comunicación e Información y la Educación.}

Las nuevas tecnologías de la Información y Comunicación (TIC), son aquellas herramientas computacionales e informáticas que procesan, almacenan, sintetizan, recuperan y presentan información representada de la más variada forma. Según Castell, (2013), al referirse a estas herramientas, menciona que estas representan canales y soportes para el tratamiento y acceso a la información, expone además, que a través de ellas, se da forma, se registra, almacena y difunde múltiples contenidos. Aludiendo el autor que algunos ejemplos de estas tecnologías son la pizarra digital (ordenador personal + proyector multimedia), los blogs, el podcast y por supuesto, la web y los wikis.

Cabe destacar, que las nuevas tecnologías no son un fin en sí mismas, sino un instrumento cuya forma de funcionar es menester aprender, para poder ser utilizadas apropiadamente como recursos para enseñar, estudiar, motivar, facilitar la comprensión, incorporar nuevos saberes y habilidades, de una manera mucho más satisfactoria, estimulando las múltiples inteligencias.

Es preciso señalar que, se cuenta hoy día en una época donde el concepto de educación y saber se invierten, pues los estudiantes suelen conocer y dominar estos enseres electrónicos y sus softwares, con absoluta suficiencia mientras muchos de los adultos que dirigen su aprendizaje miran 
para otro lado, inmovilizados por el miedo al cambio. En plena revolución de Internet, y con el conocimiento autodidacta que están adquiriendo los escolares en sus casas frente a sus ordenadores personales, el maestro se está quedando detrás del educando al no darle la utilidad en su práctica diaria en el proceso de enseñanza y aprendizaje y así brindar una mejor orientación acerca de su uso por parte del estudiante.

\section{Metodología}

La investigación se emprende con la finalidad de Generar Proposiciones Teóricas en la Formación de la Ciudadanía mediadas por la TIC en las escuelas de Educación Primaria del casco central de Santa Teresa del Tuy, todo ello, debido a que en la actualidad las Tecnologías de la Información y la Comunicación (TIC) conectivas y socioconstructivas en las comunidades virtuales, constituyen un impacto en la configuración de la ciudadanía global, ofreciendo innovación sociocultural y el cambio de mentalidades y societales.

Esta consideración lleva a enmarcar la investigación bajo el paradigma de naturaleza Cualitativa, a la cual Taylor y Bogdan (1996), refieren como aquella que se realiza desde dentro de la situación estudiada, captando la realidad de lo que dicen y hacen las personas. Además de ello, debido a la necesidad de interpretar dicha realidad, la formación de la ciudadanía bajo la configuración del uso de la tecnología se considera el carácter interpretativo, por cuanto la formación de la ciudadanía del contexto (de la globalización) aceitada telemáticamente por las TIC, se configura en un contexto inédito de saber e información, expresadas en los medios de comunicación social y en las TIC (Fainholc, 2009).

Al respecto, la investigación se basa en el enfoque de la fenomenología interpretativa de Heidegger, la cual se interesa en descubrir y entender los significados, hábitos y prácticas del ser humano, Castillo (2000: 5). Los seres humanos se encuentran en un mundo poseído de relaciones, prácticas y 
compromisos adquiridos en una cultura, de aquí que la cultura tecnológica ha influenciado en el ser humano de diversas maneras, siendo para el caso de este estudio, su influencia con el trato y las prácticas de las tecnologías de la comunicación, específicamente las redes sociales.

En la investigación se utiliza el método Comparación Constante, el cual de acuerdo con Sandoval (1997), es una continua revisión y comparación de los datos capturados para ir construyendo teoría de la realidad. La implementación de este método ayuda a que se elabore y compare nuevas categorías mentales e introduzca nociones de espacio y tiempo, de oposición y contradicción, que pueden ser extrañas al pensamiento tradicional.

En el caso de este estudio para generar Proposiciones Teóricas en la Formación de la Ciudadanía mediadas por la Tecnología de la Información y Comunicación en las escuelas de Educación Primaria, se utilizó la técnica de la entrevista que de acuerdo con Rojas (2010: 85), se define como un encuentro en el cual el entrevistado intenta obtener información, opiniones o creencias de una o varias personas.

Con respecto al instrumento a utilizar, se consideró la entrevista en profundidad, que permitió en principio a partir de preguntas Promovedoras, iniciar la conversación para a partir de allí generar nuevas preguntas que surjan a partir de la misma.

Los informantes fueron los docentes de las escuelas de Educación Primaria del casco central de Santa Teresa del Tuy, cuyas características fueron las siguientes: 1.- Docentes del 6to grado de Educación Primaria. 2.Grados que adquirieron las Canaimitas y 3.- Docentes que trabajan con el programa Canaima. Quedando conformados por cuatro (4) Docentes de tres (3) escuelas del casco central de Santa Teresa del Tuy. 
Para analizar los resultados obtenidos en las entrevistas, en el caso, del procedimiento de la Teoría Fundamentada, el análisis plantea tres fases:

Etapa 1. Codificación abierta, de acuerdo con el autor mencionado anteriormente supone un proceso de involucramiento con la realidad objeto de estudio para comenzar a recolectar la información por diversas vías, de acuerdo con los requerimientos de la investigación. En esta fase se inicia el proceso de construcción de categorías que pueden surgir de los sujetos in vivo, pero también de la elaboración conceptual y teórica realizada por el investigador.

Etapa 2. Codificación Axial, Se identifican las relaciones entre las categorías y las subcategorías, lo cual puede llevar a modificar la estructura inicial, se inicia aquí la generación de teoría y su validación con los datos. Esta generación de teoría se produce cuando se dan las respuestas a al cuándo, dónde, por qué, qué consecuencias se tienen del fenómeno detectado.

Etapa 3. Codificación Selectiva, se caracteriza por la identificación de las categorías principales, más inclusivas y la modificación de la estructura en función de las mismas. Se trata de integrar y refinar las categorías. Comienza la producción del texto narrativo y continúa la validación de la teoría para su presentación final. Cabe destacar, que la investigadora se planteó diversas fases a seguir, para un orden lógico, de acuerdo con las necesidades del estudio:

Fase I. Detección del Objeto de Estudio y Arqueo de la Información.

Determinación de la investigación: estará comprendida por la aproximación al planteamiento del problema, los objetivos y justificación, así como también, las estrategias que contribuirán a realizarlo y concretarlo.

Fase II. Organización de la Información teórica para la construcción del Estado del Arte.

En esta fase a partir de la concreción del objeto de estudio, y la revisión bibliográfica desarrollada, se organiza y clasifica el material bibliográfico 
relacionado con la temática, identificando los antecedentes y las bases teóricas.

Fase III. Búsqueda de la Información en el Campo.

Constituye la realización de los tres (3) momentos de interacción para la búsqueda de la información: Momento 1: acceso al campo y condiciones de confianza. Momento 2: Presentación de la entrevista. Momento 3: Validación de la información obtenida a través de la recapitulación.

Fase IV. Entramado Analítico.

Esta fase implica que el investigador utilice diversos métodos o procedimientos para organizar la información que le permita construir las codificaciones. Entre los procedimientos a considerar se tienen: Descargar la información y asentarla en tablas que permitan visualizar para leer y releer la información obtenida. Selección de unidades de análisis heurísticas, es decir, ofrecer información relevante para el estudio y estimular la comprensión del lector acerca del problema estudiado. Cada unidad de análisis debe contener tantas categorías y subcategorías como surjan en el proceso de análisis.

Fase V. Construcción de la Teoría.

Esta fase permite la reconstrucción de la realidad a partir de los hallazgos encontrados.

A continuación, se detallan los resultados obtenidos como producto de las entrevistas a los informantes clave. Los mismos se realizan en función de citas parciales a fin de lograr interpretar el significado de las Tecnologías de la Información y Comunicación (TIC) en el proceso de formación ciudadana, considerando así, la idea de Sanmartín (2003: 105), quien expresa que: "En la redacción que finalmente construimos constan citas parciales de lo registrado en las entrevistas".

Para la comprensión de los datos obtenidos, se construyeron bloques temáticos que surgen de la triangulación de las respuestas dadas por los 
docentes entrevistados:

(a) Fin educativo del Programa Canaima. El fin educativo de este programa es incluir herramientas tecnológicas dentro del aula para lograr el alcance de la albanización tecnológica desde la educación primaria y brindar una orientación educativa en estos medios y la utilidad por parte del escolar.

(b) Canaima como herramienta en el aula. Esta herramienta permite al docente la implementación de un ambiente de aprendizaje tecnológico, moderniza las estrategias del docente e incentiva la adquisición de la modernización en el aula y el conocimiento.

(c) Proceso de socialización y valores en los escolares. Este proceso de socialización comienza en la familia, se da en las iglesias y continúa en la escuela y ahora las redes sociales ejercen influencia en ella, ya que los individuos se han socializado de manera significativa a través de las mismas. Por tanto, se requiere de un proceso de formación de padres y maestros para que comiencen a supervisar este proceso de socialización tan moderno.

(d) Formación Ciudadana en los escolares. La formación del ciudadano se inicia en el hogar y en la comunidad, la escuela lo reafirma y lo orienta, no obstante, las redes sociales están interviniendo en esta formación, mediante todos los contenidos que se comparten en las redes del internet. Es necesario que los adultos, padres y educadores tomen parte en lo planteado para canalizar y orientar en valores, así como crear conciencia de lo que observan los escolares y valerse de ello para formar en valores, educación sexual e identidad y formar en ciudadanía a través de estas herramientas.

\section{Hallazgos}

Este apartado está orientado a mostrar algunos hallazgos encontrados como producto del análisis realizado a lo largo de la investigación. Para ello, y su mejor comprensión se plantean estos en el mismo orden de los propósitos específicos planteados y en función de dar respuestas a las interrogantes 
expuestas por la investigadora.

Interrogante 1. ¿Cuáles son las metodologías pedagógicas insertas en las Tic utilizadas por los docentes de las escuelas de educación primaria del casco central de Santa Teresa del Tuy al orientar la formación de la ciudadanía en los educandos?

1.- Aprovechamiento de las conversaciones informales de los estudiantes para abordar cualquier temática que se presente ajena al contenido planificado.

2.- Actividades de reforzamiento en el desarrollo de cualquier contenido.

3.- Actividades relacionadas con el proyecto de aprendizaje que permitan utilizar la Canaima.

4.- La investigación como estrategia para el desarrollo de temas tanto de las TICS como de la Formación Ciudadana.

5.- Actividades de investigación que permitan al estudiante adquirir nuevos conocimientos.

6.- Sustitución del cuaderno y lápiz por el uso del computador (Canaima) para el máximo aprovechamiento del recurso, de esta manera aprenden a valorar y cuidar su equipo.

En torno al segundo propósito, que lleva a la interrogante ¿Cómo ha sido la orientación educativa que asumen los docentes de las escuelas de educación primaria del casco central de Santa Teresa del Tuy ante la utilización de la Tecnología de la Información y Comunicación para la formación de la ciudadanía de los escolares?

1.- Proceso de formación continua y de avanzada hacia los docentes, quienes son los que van a orientar el proceso de enseñanza y aprendizaje de sus estudiantes.

2.- Seguimiento permanente al uso y manejo del Programa Canaima. 
Con respecto al tercer propósito específico que orienta hacia ¿Cuál es el significado que le asignan los docentes de las escuelas de educación primaria, al uso de las Tecnologías de la Información y Comunicación en el proceso de formación ciudadana en los escolares de estas escuelas?, se puede señalar que se interpreta hacia la relación directa de los valores, considerando que el uso de las TICS, tiene sus ventajas y desventajas, entre las primeras (ventajas), se pueden señalar las siguientes:

1.- Desde el punto de vista cultural, se adquieren aprendizajes de diversas culturas ampliando el repertorio del conocimiento con relación a contextos sociales y culturas diferentes.

2.- La formación ciudadana como forma de adquisición de normas y convivencia, debe ser respetada en el contexto de las redes sociales, la participación del estudiante y el uso del Facebook como estrategia de enseñanza debe partir por el respeto al otro y a todos quienes visualizan el contenido guindado, ya que éste es un medio que se encuentra al alcance de todos.

3.- El Programa Canaima brinda la posibilidad al estudiante de desarrollar habilidades y destrezas en diversas áreas del aprendizaje.

En cuanto a las desventajas, se pueden señalar:

1.- La inserción de la planificación del Programa Canaima ha sido impositiva y obligada.

2.- La falta de seguimiento o articulación con la realidad social imposibilitan el trabajo con las TICS, motivado a las debilidades presentes en el programa, tales como: (a) la no asignación de computadores a los docentes; (b) la pérdida sin reposición del equipo a los niños. (c) El no contar con un centro de reparación o canje, que permita llevar la mini laptop por presentar cualquier daño o deterioro técnico. 
Finalmente, el propósito específico 4, referido a la interrogante ¿Qué orientaciones pueden surgir a través de esta indagación para la generación de proposiciones teóricas en la Formación de la Ciudadanía mediante la Tecnología de la Información y Comunicación?, se puede indicar que muchas son las ideas que emergieron en este desarrollo, sin embargo, se centró la atención en los siguientes enunciados:

1.- La necesidad del empoderamiento por parte de los docentes en el uso de las TICS que lo llevan a utilizar el Programa Canaima en el aula.

2.- Promoción de la importancia de un buen ciudadano a través de la reflexión de temas que se orienten a investigar en la web.

3.- Diversificación y ampliación del abanico de estrategias que permitan la utilización de videos, la práctica cooperativa, a la colaboración e intercambio de información entre los pares a través de las redes sociales.

4.- Promoción de un Centro de Reparación, Préstamo e Intercambio de Canaima (CENREPREINCA), para agilizar el mantenimiento preventivo y correctivo de los equipos.

\section{Proposiciones Teóricas.}

1). La Ciudadanía Tecnológica depende no solo de la inversión de recursos sino de la formación en valores como responsabilidad y compromiso.

Este postulado teórico representa un aspecto fundamental en el desarrollo del proceso de formación de ciudadanía, considerando como valores básicos la responsabilidad y el compromiso, el primero como referencia al uso adecuado de las redes sociales; y el segundo, a la adquisición del conocimiento que lleve a la transformación social.

La educación transformadora busca integrar desde el humanismo la relación docente - estudiante dando un acercamiento al aprendizaje desde el respeto, el amor y la comprensión del hombre hacia sí mismo y del hombre hacia el hombre. Freire (2004), refiere que la educación transformadora busca 
ser práctica de la libertad hacia la transformación del mundo.

Considerando que las TIC son la base para las nuevas relaciones con las personas en un esquema de tiempo - espacio - vínculo, los centros educativos se han convertido en escenarios, no solo del aprendizaje de la lengua, la matemática y otras áreas del saber, ahora con el uso del computador en el aula, son los encargados de generar el conocimiento, construir y reconstruirlo de manera cónsona con la actual sociedad.

2). La escuela no es el único ambiente de aprendizaje, el espacio tecnológico que ofrece la Canaima brinda un sinfín de oportunidades para el aprendizaje colaborativo en la red.

La escuela es por tradición el lugar para el aprendizaje, la socialización y formación de los niños y niñas, ha representado un aspecto significativo en el desarrollo integral que junto con la familia constituye el espacio para la convivencia y la formación de la ciudadanía. Durkheim (1976), refiere que la escuela "es un lugar donde además de preparar a los individuos para que hagan parte de la sociedad que los ha acogido, los responsabiliza de su conservación y de su transformación".

Un ambiente de aprendizaje constituye el espacio, las estrategias, las herramientas, que puede ser utilizado de forma amena para el logro de objetivos planificados, los ambientes pueden clasificarse en áulico, físico y virtual, el tercero, más cercano a la modernidad, se realiza con el uso del internet, a través de las diversas herramientas que ofrece la navegación, los blogs, correos electrónicos, aulas virtuales, y que permiten compartir, discutir y aportar informaciones para la reflexión y el aprendizaje.

Bajo este contexto, los espacios virtuales de aprendizaje, que pueden ser utilizados en el Programa Canaima va a la Escuela, o Canaima va a la Casa, ofrece gran variedad de oportunidades para compartir y aprender, entre ellas, se pueden mencionar: 
1.- A partir de videos vistos en el hogar, se pueden realizar diversas reflexiones sobre un tema en particular, en el caso de la formación ciudadana, pueden ofrecerse cortos con experiencias de diversos tipos, y permitir a los estudiantes, opinar y buscar alternativas de solución a los planteamientos realizados.

2.- La investigación sobre temas específicos, para construir resúmenes y conclusiones.

3.- La participación en blogs escolares, que generen opiniones y reflexiones sobre temas particulares del comportamiento de las personas.

4.- El trabajo cooperativo para la construcción de cuentos, partiendo de una idea inicial y cada estudiante va agregando situaciones a ella.

5.- Compartir mensajes de motivación, respeto y convivencia a través del Facebook.

6.- Creación de radios escolares, periódicos infantiles, competencias sanas, de construcciones como diccionarios virtuales, entre otros.

3). El docente tiene una función fundamental y un reto que afrontar en la formación de una ciudadanía y cultura tecnológica.

La escuela no solo basa su evolución en el proceso de enseñanza de las letras y contenidos programados de acuerdo a la edad y el grado, es una institución que permite la construcción colectiva de las normas, valores y principios para y desde la convivencia, de tal manera que es forjadora de elementos fundamentales para asumir de manera responsable la enseñanza de la democracia y la participación ciudadana, de tal manera que constantemente debe ofrecer la enseñanza de un buen ciudadano, en el contexto social tradicional, y ahora con el uso de las Tecnologías de la Información y la Comunicación, el comportamiento ajustado al respeto en las diferentes redes sociales. 


\section{Conclusión.}

Para finalizar, es importante hacer referencia a lo descrito por Martínez (2016), en cuanto a: "El docente debe asumir su nuevo rol dentro del proceso educativo, a fin de brindar una educación de calidad. La formación inicial y permanente del profesorado en materia de tecnología es fundamental para garantizar su adecuación al entorno educativo en el cual deberá desarrollar su tarea docente". (pág. 102).

Al respecto, las proposiciones teóricas presentadas vislumbran la tarea que asumen los docentes de hoy, a la cual hace referencia el autor antes citado en cuanto la constante formación que es un reto en su carrera, ya que las TIC se convirtieron en un medio de vida de los ciudadanos, en especial de los más pequeños que están en formación. Por tal motivo, se hace necesaria la puesta en prácticas de estrategias innovadoras que puedan dar un giro a la enseñanza sin orientación educativa que les está proporcionando la tecnología moderna a los ciudadanos.

\section{Referencias}

Castell, M. (2013). Comunicación y Poder. Redes, Tecnología y Educación. España: Alianza Editorial.

Castillo, E. (2000). La fenomenología interpretativa como alternativa apropiada para estudiar los fenómenos humanos. Investigación y Educación en Enfermería. vol. XVIII, núm. 1, marzo, 2000, págs. 27-35, Colombia: Universidad de Antioquia. Recuperado de:

http://www.redalyc.org/pdf/1052/105218294002.pdf

Durkheim, E. (1976). Educación como socialización. Salamanca: Ediciones Sígueme.

Fainholc, C. (2009). Pensar una ciudadanía para la sociedad del conocimiento con la formación y práctica del socio constructivismo crítico de las TICs. Toluca, México. Recuperado de: 
http://132.248.9.34/hevila/Tecnologiaycomunicacioneducativas/2008-

09/vol22/no47-48/4.pdf

Freire, P. (2004). La Importancia de leer y el Proceso de liberación. Buenos aires: siglo XXI, Ediciones Argentina. S.A.

Horrach, J. (2009). Sobre el concepto de ciudadanía: historia y modelos. España: Universidad de las Islas Baleares.

Hoyuelos, A. (2006). La estética en el pensamiento y obra pedagógica de Loris Malaguzzi. Barcelona: Octaedro-Rosa Sensat.

Luján, M., \& Salas F. (2009). Enfoques Teóricos y definiciones de la Tecnología Educativa en el Siglo XX. Revista Electrónica "Actualidades Investigativas en Educación". Recuperado de:

http://www.redalyc.org/html/447/44713058004/

Martínez, O. (2016). Programa de Formación Docente de las Tecnologías del Aprendizaje y el Conocimiento (Tac) en la Universidad Pedagógica Experimental Libertador Núcleo Barinas (Venezuela). Revista Scientific, 1(1), 90-114. Recuperado de:

https://doi.org/10.29394/scientific.issn.2542-2987.2016.1.1.6.90-114

Morawietz, L. (2015). Aprendizajes para el ejercicio de la ciudadanía. Centro de Investigación Avanzada en Educación, Universidad de Chile. Recuperado de:

http://www.unesco.org/fileadmin/MULTIMEDIA/FIELD/Santiago/pdf/APU NTE07-ESP.pdf

Rojas, B. (2010). Investigación Cualitativa. Fundamentos y Praxis. Caracas: FEDUPEL.

Rueda, Y. (2009). Innovación para el empoderamiento de la Ciudadanía a través de la TIC. Madrid: España.

Sandoval, C. (1997). Investigación cualitativa. módulo 4. programa de especialización en teoría, métodos y técnicas de investigación social. universidad de Antioquía. Medellín: Ascun, pág. 433. 
Sanmartín, R. (2003). Observar, Escuchar, Comparar, Escribir. La práctica de la Investigación Cualitativa. Barcelona, España: Ariel.

Taylor, S. \& Bogdan R. (1996). Introducción a los Métodos Cualitativos de la Investigación. México: Paidos. 


\section{Tania Margarita Martínez de Padrón}

e-mail: tania.martinez10@gmail.com

Nacida en Venezuela. Licenciada en Educación egresada

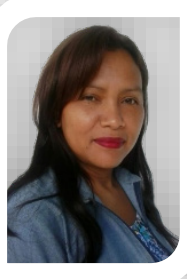
de la Universidad Nacional Experimental Simón Rodríguez (UNERS). Especialista en Planificación y Evaluación (UNERS). Diplomado en Investigación. Universidad Nacional Experimental de la Fuerza Armada Nacional Bolivariana. UNEFA. Magister Scientiarum en Tecnología Educativa. Universidad Nacional Experimental de la Fuerza Armada Nacional Bolivariana. UNEFA. Doctorante en Ciencias de la Educación Universidad Latinoamericana y del Caribe (ULAC).

Subdirectora y Docente de Aula en ejercicio de la: U.E.E. "La cruz". Docente de Postgrado de la Universidad Nacional Experimental de la Fuerza Armada Nacional Bolivariana (UNEFA). Tutora de Tesis de Postgrado (UNEFA).

El contenido de este manuscrito se difunde bajo una Licencia de Creative Commons Reconocimiento- 